\title{
Subgrid parameterisations for primitive equation atmospheric models
}

\author{
J. S. Frederiksen ${ }^{1} \quad$ M. R. Dix ${ }^{2} \quad$ S. L. Osbrough ${ }^{3}$ \\ V. Kitsios ${ }^{4}$
}

(Received 27 February 2015; revised 13 October 2015)

\begin{abstract}
Dynamical and thermodynamical subgrid-scale parameterisations of eddy drain, net dissipation and stochastic backscatter are calculated for a multi-level primitive equation atmospheric general circulation model. The parameterisations have only moderate variability with height and a cusp behaviour with peaks near the largest retained wavenumber. Vertically integrated net dissipation functions for vorticity and temperature are very similar to corresponding results for barotropic simulations while the divergence dissipation is nearly four times stronger. Atmospheric general circulation model simulations with the new subgrid model improve kinetic energy spectra and zonal flows compared with control simulations.
\end{abstract}

http://journal.austms.org.au/ojs/index.php/ANZIAMJ/article/view/9358 gives this article, (C) Austral. Mathematical Soc. 2015. Published November 5, 2015, as part of the Proceedings of the 17th Biennial Computational Techniques and Applications Conference. ISSN 1446-8735. (Print two pages per sheet of paper.) Copies of this article must not be made otherwise available on the internet; instead link directly to this URL for this article. 


\section{Contents}

1 Introduction

C84

2 Primitive equation spectral model

C85

3 Stochastic modelling technique

C86

4 Primitive equation subgrid model

C87

5 AGCM simulations with subgrid model

C93

6 Conclusions

C98

References

C99

\section{Introduction}

Since the first climate simulations with atmospheric general circulation models (AGCMs) it was found that parameterisations of subgrid-scale processes strongly influence the accuracy of the large-scale flows and energy spectra [5]. As reviewed by Frederiksen et al. [4], in recent years there has been considerable progress in solving this long standing problem using systematic and self consistent closure, and stochastic modelling approaches. For both atmospheric and oceanic flows described by quasi-geostrophic equations, universal scaling laws for the subgrid eddy dissipation were derived [7] and the stochastic modelling approach was also successfully applied to three-dimensional turbulence in boundary layer channel flows [8]. The aim of this article is to apply the method of Frederiksen and Kepert [3] to determine parameterisations of subgrid eddy-eddy interactions for the more complex primitive equation atmospheric models. Section 2 summarises the spectral primitive equations for atmospheric flows. The stochastic modelling technique for determining 
subgrid-scale parameterisations is summarised in Section 3. Eddy dissipation and stochastic backscatter terms are calculated in Section 4. Vertically averaged subgrid terms are also described in Section 4 and are applied to the primitive equation AGCM in Section 5. Comparisions of the results with observations and control simulations with a standard heuristic eddy diffusion model are also presented in Section 5.

\section{Primitive equation spectral model}

The simulations and studies in this article were performed with the CSIRO 9-level AGCM [9]. It is a sigma coordinate model where $\sigma=p / p_{s}, p$ is the pressure and $p_{s}$ is the the surface pressure. The prognostic variables are surface pressure weighted: $\zeta=p_{s} \xi, T=p_{s} \mathcal{T}, D=p_{s} \mathcal{D}$, and $p_{s}$. Here, $\xi$ is vorticity, $\mathcal{T}$ is temperature and $\mathcal{D}$ is divergence. These variables are expanded in terms of spherical harmonics and the spectral coefficients satisfy the thermodynamical equations

$$
\begin{aligned}
\frac{\partial \zeta_{m n}}{\partial t} & =N_{m n}^{\zeta}+f_{m n}^{\zeta}(t)-D_{0}^{\zeta}(n, \sigma) \zeta_{m n}, \\
\frac{\partial D_{m n}}{\partial t} & =N_{m n}^{D}+f_{m n}^{D}(t)-D_{0}^{D}(n, \sigma) D_{m n}, \\
\frac{\partial T_{m n}}{\partial t} & =N_{m n}^{T}+f_{m n}^{T}(t)-D_{0}^{\top}(n, \sigma)\left[T_{m n}-T_{m n}^{e q}\right], \\
\frac{\partial p_{s m n}}{\partial t} & =N_{m n}^{p}+f_{m n}^{p}(t)-D_{0}^{p}(n, \sigma) p_{s m n} .
\end{aligned}
$$

Here $m$ is the zonal wavenumber, $n$ the total wavenumber, $D_{0}^{\bullet}$ are the specified (bare) dissipation-dispersion functions, $\mathbf{f}_{\mathrm{mn}}^{\bullet}$ are the dynamical and thermodynamical forcings and $\mathrm{N}_{\mathrm{m} n}^{\bullet}$ are nonlinear terms. Also, $\mathrm{T}_{\mathrm{mn}}^{e q}=[\overline{\mathrm{T}}+$ $\left.\sigma \frac{\partial \overline{\mathrm{T}}}{\partial \sigma}-\mathrm{T}_{0}\right] p_{\text {smn }}$ where $\overline{\mathrm{T}}$ is the global mean $\sigma$-level temperature and $\mathrm{T}_{0}=290 \mathrm{~K}$. The nonlinear terms are quadratic in the prognostic variables. We define $\mathrm{k}=(\mathrm{m}, \mathrm{n})=\left(\mathrm{m}_{\mathrm{k}}, \mathrm{n}_{\mathrm{k}}\right)$ where $-\mathrm{k}=(-\mathrm{m}, \mathrm{n})$ and $\mathrm{q}_{\mathrm{k}}^{\mathrm{a}}=\left(\zeta_{\mathrm{k}}^{\mathrm{j}}, \mathrm{D}_{\mathrm{k}}^{\mathrm{j}}, \mathrm{T}_{\mathrm{k}}^{\mathrm{j}}, \mathrm{p}_{\mathrm{sk}}\right)$ 
where $a=1, \ldots, 28$ and $j=1, \ldots, 9$ is the $\sigma$-level. That is, $a=j$ for $q=\zeta$, $a=9+j$ for $q=D, a=18+j$ for $q=T$, and $a=28$ for $q=p_{s}$. Then the nonlinear terms are

$$
N_{k}^{a}=\sum_{p} \sum_{q} K^{a b c}(k, p, q) q_{-p}^{b}(t) q_{-q}^{c}(t)
$$

where the summation is implied over repeated superscripts [1] and are over the triangular truncation set

$$
\mathbf{T}(\mathrm{T})=\left\{\mathbf{p}, \mathbf{q}\left|-\mathrm{T} \leqslant \mathrm{m}_{\mathrm{p}} \leqslant \mathrm{T},\right| \mathrm{m}_{\mathrm{p}}\left|\leqslant \mathrm{p} \leqslant \mathrm{T},-\mathrm{T} \leqslant \mathrm{m}_{\mathrm{q}} \leqslant \mathrm{T},\right| \mathrm{m}_{\mathrm{q}} \mid \leqslant \mathrm{q} \leqslant \mathrm{T}\right\} .
$$

Also, $\mathbf{K}^{\mathrm{abc}}$ are interaction coefficients between the nonlinear terms in equation (2).

\section{Stochastic modelling technique}

We now consider the subgrid model required to compensate for reducing the resolution from that of the benchmark simulation equation (3) to a large eddy simulation (LES) with resolution $\mathbf{R}=\mathbf{T}\left(T_{R}\right)$. Here $T_{R}$ is the LES truncation wavenumber and $T>T_{R}$. The subgrid wavenumber set is defined as $\mathbf{S}=\mathbf{T}-\mathbf{R}$. We employ the stochastic modelling approach described by Frederiksen and Kepert [3] to formulate the subgrid model. Let $\mathbf{q}$ denote the column vector with components $q_{m n}^{a} \equiv q_{k}^{a}$ where a runs over the 28 field variables. Then the prognostic equation (1) is

$$
q_{t}(t)=q_{t}^{R}(t)+q_{t}^{S}(t)=q_{t}^{R}(t)+\bar{f}+\widehat{q}_{t}^{S}(t)
$$

where $\mathbf{q}_{\mathbf{t}}^{\mathbf{R}}(\mathbf{t})$ is the tendency of the retained scales, and $\mathbf{q}_{\mathbf{t}}^{\mathbf{S}}(\mathbf{t})$ is the subgrid tendency consisting of the time averaged component $\overline{\mathbf{f}}=\overline{\mathbf{q}}_{\mathbf{t}} \mathbf{S}(\mathrm{t})$ and the fluctuating component $\widehat{\underline{\mathbf{q}}}_{\mathrm{t}}^{\mathbf{s}}(\mathrm{t})$. In the current study where baroclinic instability is resolved in the LES, $\overline{\mathrm{f}} \approx 0$. 
The fluctuating component of the subgrid tendency is

$$
\widehat{\mathbf{q}}_{t}{ }_{t}(t)=-D_{d} \widehat{\mathbf{q}}(t)+\widehat{\mathbf{f}}(t),
$$

where $D_{d}$ is the subgrid drain dissipation matrix, $\widehat{\mathbf{q}}$ is the fluctuating component of $\mathbf{q}$, and $\widehat{\mathbf{f}}$ is a random forcing vector. A generalisation of Gauss' theorem of least squares $[3,7]$ then yields

$$
D_{d}=-\left\langle\int_{t_{0}}^{t} \widehat{\mathbf{q}}_{t}^{s}(s) \widehat{\mathbf{q}}^{\dagger}\left(t_{0}\right) d s\right\rangle\left\langle\int_{t_{0}}^{t} \widehat{\mathbf{q}}(s) \widehat{\mathbf{q}}^{\dagger}\left(t_{0}\right) d s\right\rangle^{-1},
$$

where $\dagger$ denotes the Hermitian conjugate. With $D_{d}$ determined by equation (6), the Lyapunov or balance equation

$$
\left\langle\widehat{\mathbf{q}}^{\mathbf{S}}{ }_{t}(\mathrm{t}) \widehat{\mathbf{q}}^{\dagger}(\mathrm{t})\right\rangle+\left\langle\widehat{\mathbf{q}}(\mathrm{t}) \widehat{\mathbf{q}}^{\mathrm{S}^{\dagger}}{ }_{\mathrm{t}}(\mathrm{t})\right\rangle=-\mathrm{D}_{\mathrm{d}}\left\langle\widehat{\mathbf{q}}(\mathrm{t}) \widehat{\mathbf{q}}^{\dagger}(\mathrm{t})\right\rangle-\left\langle\widehat{\mathbf{q}}(\mathrm{t}) \widehat{\mathbf{q}}^{\dagger}(\mathrm{t})\right\rangle \mathrm{D}_{\mathrm{d}}^{\dagger}+\mathcal{F}_{\mathrm{b}},
$$

specifies the covariance of the random forcing $\mathcal{F}_{b}=F_{b}+F_{b}^{\dagger}$ where $F_{b}=$ $\left\langle\widehat{\mathbf{f}}(\mathrm{t}) \widehat{\mathbf{q}}^{\dagger}(\mathrm{t})\right\rangle$. We also define backscatter and net dissipation operators by $D_{b}=-F_{b}\left\langle\widehat{\mathbf{q}}(t) \widehat{\mathbf{q}}^{\dagger}(\mathrm{t})\right\rangle^{-1}$ and $D_{n}=D_{d}+D_{b}$, respectively, and corresponding eddy viscosities by $v_{\bullet}=D_{\bullet} / n(n+1)$.

\section{Primitive equation subgrid model}

We use the techniques of Section 3 to calculate stochastic and deterministic subgrid scale parameterisation based on simulations of the primitive equation AGCM described in Section 2. For this simulation we employ the simple relaxation physics forcing described by Held and Suarez [6]. We are interested in obtaining generally applicable subgrid models that can be used over the seasonal cycle. The diffusion function employed in this simulation is based on the barotropic subgrid model studies of Frederiksen and Davies [2] and Frederiksen and Kepert [3].

Figure 3(d) of Frederiksen and Kepert [3] shows the isotropised net dissipation function required to make barotropic LES at $\mathrm{T} 31\left(\mathrm{~T}_{\mathrm{R}}=31\right)$ have the same 
Figure 1: Net eddy dissipation function $g\left(n / n_{*}\right)$ for the relaxation physics model.

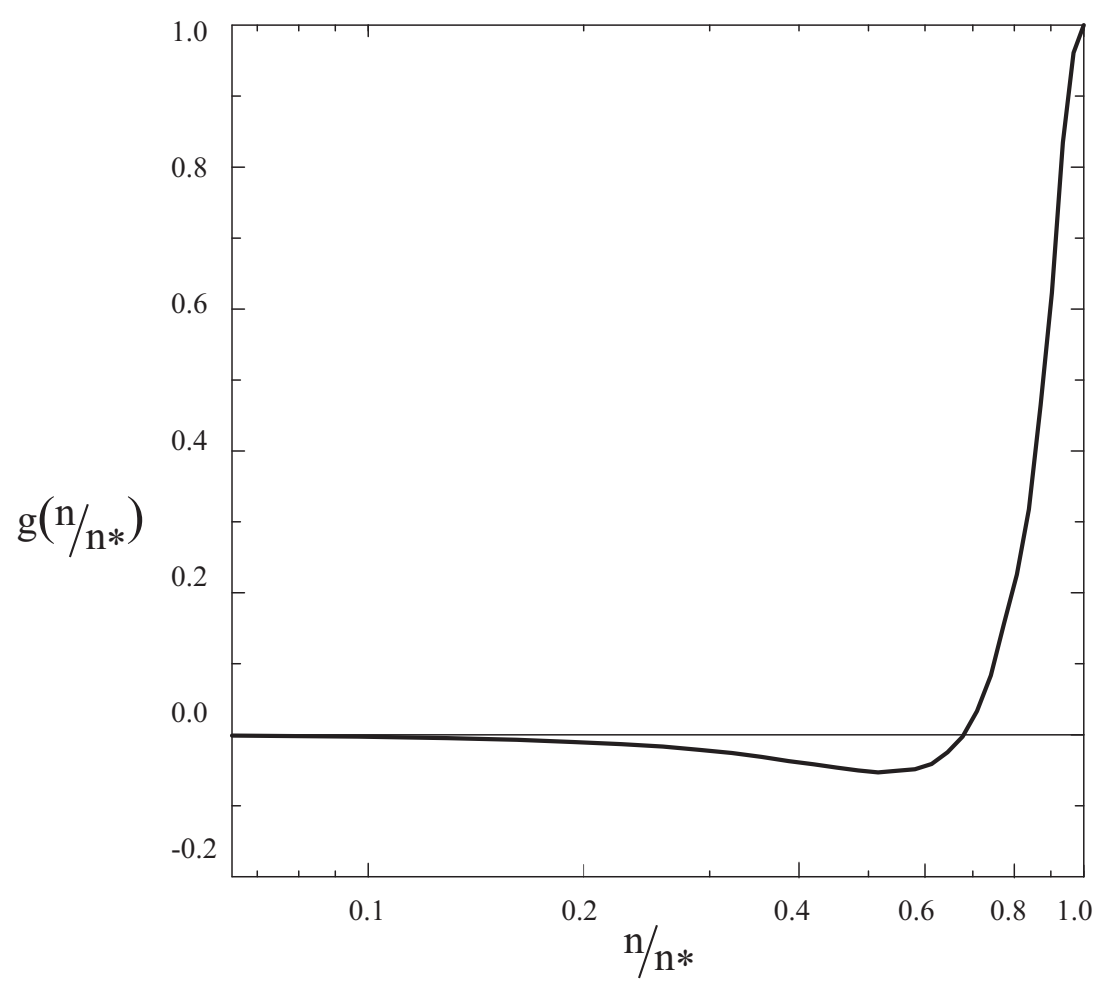

resolved scale spectra as the higher T63 $(T=63)$ resolution benchmark direct numerical simulation (DNS). This same net dissipation function $g\left(n / n_{*}\right)$ is shown in Figure 1, scaled to a peak of unity and as a function of scaled wavenumber $\left(n / n_{*}\right)$ where $n_{*}$ is the maximum resolution. The cusp shape near the maximum wavenumber is a general characteristic of net dissipation functions. We now examine the performance of the model when employed for $n_{*}=63$. The net dissipation and diffusion coefficients are

$$
D_{0}^{\bullet}(n, \sigma)=v_{0}^{\bullet} n_{*}\left(n_{*}+1\right) g\left(n / n_{*}\right) .
$$

Here $\bullet \in[\zeta, D, T, p]$ and $v_{0}^{\zeta} a_{e}^{2}=2.46 \times 10^{5} \mathrm{~m}^{2} \mathrm{~s}^{-1}$ where $a_{e}$ is the earth's 
Figure 2: Monthly averaged kinetic energy spectra $e(n)\left(m^{2} s^{-2}\right)$ for the relaxation physics model.

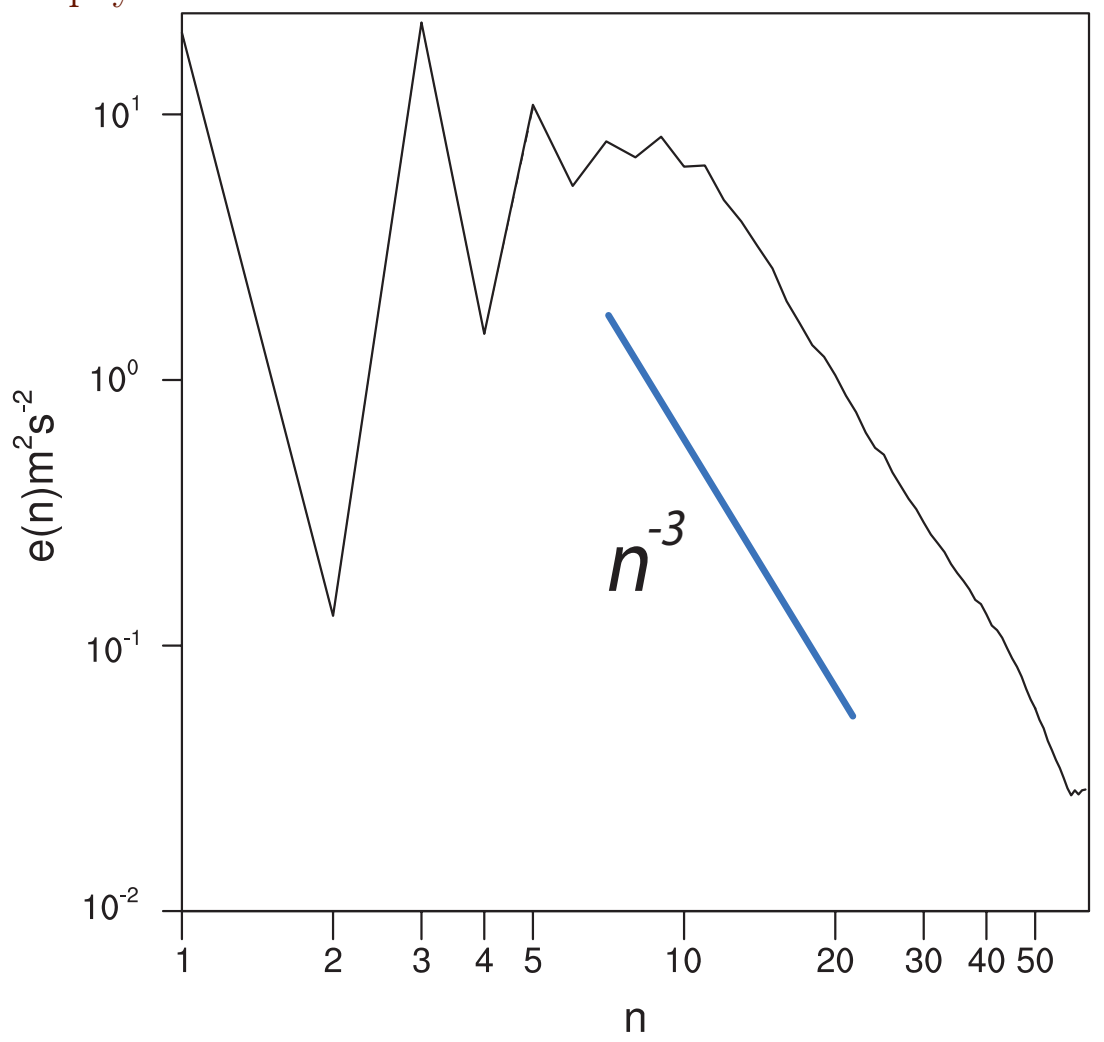

radius $\left(6.371 \times 10^{6} \mathrm{~m}\right)$. We use $v_{0}^{\top}=v_{0}^{\zeta}$ and $v_{0}^{\mathrm{D}}=4 v_{0}^{\zeta}$. We also define $\mathrm{g}^{+}\left(\mathrm{n} / \mathrm{n}_{*}\right)=\mathrm{g}\left(\mathrm{n} / \mathrm{n}_{*}\right)$ when $\mathrm{g}$ is positive but $\mathrm{g}^{+}$is zero when $\mathrm{g}$ is negative. We examine the performance of both functions in Section 5 but for the simulations in this section we replace $\mathrm{g}$ by $\mathrm{g}^{+}$.

A long spin up run of the model was performed and the kinetic energy spectra and circulation features, particularly zonal winds, were examined. Figure 2 shows the vertically integrated kinetic energy spectrum averaged over the last three months of the simulation. The total wavenumber spectrum has an 
Figure 3: Monthly and zonally average zonal wind cross-sections as functions of latitude and pressure for (a) AGCM with relaxation physics, (b) January observations, (c) January AGCM control run, (d) January AGCM S10 run with new subgrid model.

(a)

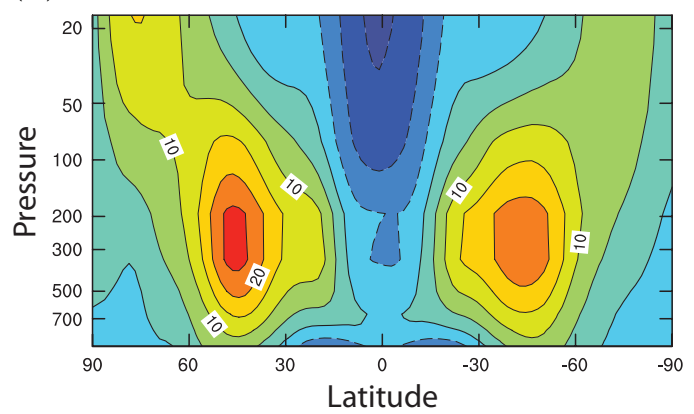

(c)

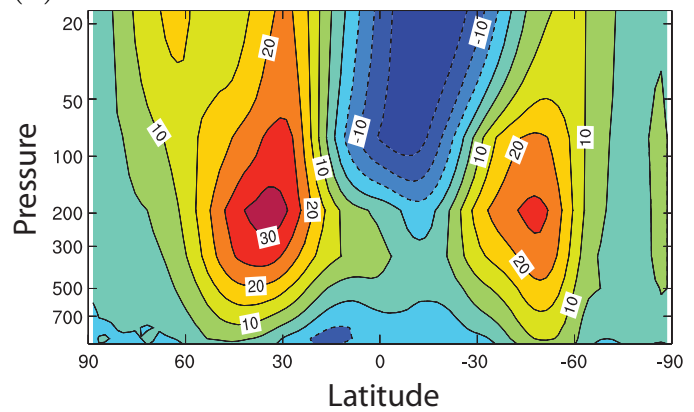

(b)

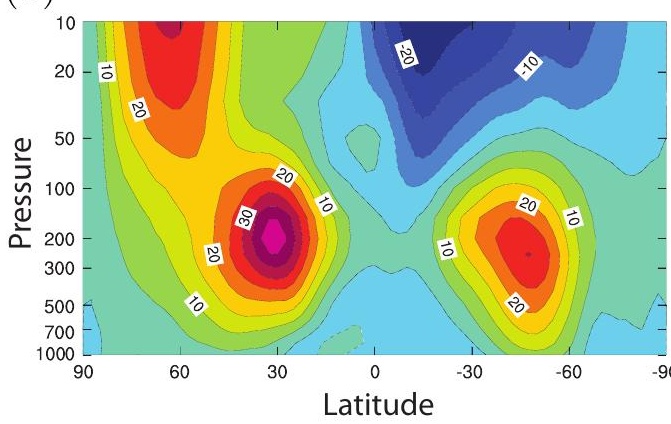

(d)

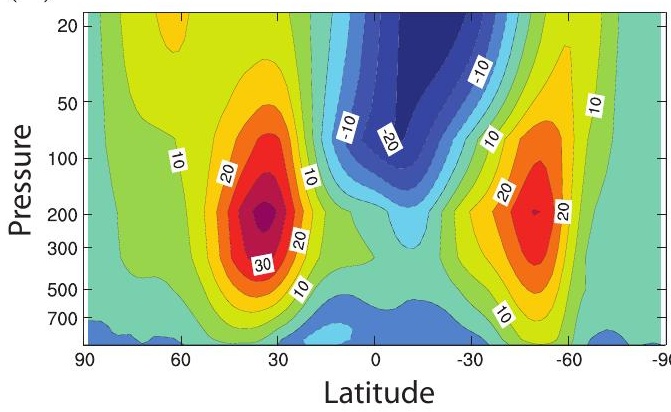

approximate $n^{-3}$ inertial range between $n=15$ and $n=40$, and then falls off slightly faster towards the largest wavenumbers. Figure 3(a) shows the structure of the zonal wind, averaged over the last month of simulation; peak jet streams occur in the upper troposphere of both hemispheres centred near $45 \mathrm{~N}$ and $45 \mathrm{~S}$. The zonal winds are more characteristic of the annual average flow than those for a particular month, such as the January observations in Figure 3(b).

Next, we apply the stochastic modelling approach of Section 3 to derive 
stochastic and deterministic net parameterisations of subgrid eddy-eddy interactions when the model resolution is reduced from T63 to T31. We employ the methodology of Section 3 for each variable at a given level and wave number pair $(m, n)$ using a time interval of four days in equation (6). We then average over the zonal wave number $m$ to obtain isotropised coefficients. Figures 4 and 5 show the resulting nondimensional drain, backscatter and net dissipation functions for the vorticity as well as the net divergence, temperature and surface pressure dissipation functions. We use $a_{e}$ (earth's radius) and $\Omega^{-1}$ (inverse of earth's angular velocity) as length and time scales. The drain and particularly backscatter dissipation functions for vorticity are essentially equivalent barotropic (uniform in the vertical $\sigma$ direction) with a cusp behaviour maximising near the largest retained wave number $n=31$. The net dissipation for vorticity varies somewhat more with height, with maxima near the surface and $300 \mathrm{hPa}$. This is also seen for the net temperature diffusion while the net divergence dissipation maximum occurs near the surface and at the highest retained wavenumber $n=31$. The net dissipation for the surface pressure has a qualitatively similar structure to the barotropic function $\mathrm{g}\left(\mathrm{n} / \mathrm{n}_{*}\right)$ but with smaller negative contribution.

The vertical average of the net dissipation function for vorticity (Figure 4(c)) is very similar to $\mathrm{g}$ in Figure 1 and it has a peak nondimensional value $\approx 0.1$ (not shown). That is, in both structure and strength it is essentially the same as the barotropic curve Figure 3(d) of Frederiksen and Kepert [3]. Similarly, the vertical average of the net dissipation or diffusion function for temperature closely follows the cusp behaviour of $g$ with a peak nondimensional value of $\approx 0.08$ (not shown) but like $\mathrm{g}^{+}$has no negative contribution.

The vertical average of the net dissipation function for divergence (not shown) is cusped at the truncation wavenumber $n_{*}=31$ but without the negative contribution of the vorticity diffusion function. At the peak its value is $\approx 0.35$ in nondimensional units, or nearly four times that of the vorticity and temperature diffusion functions. Thus, choosing $v_{0}^{\mathrm{D}}=4 v_{0}^{\zeta}$ in equation (8) and $\kappa_{0}^{\mathrm{D}}=4 \kappa_{0}^{\zeta}$ in equation (9) below is broadly consistent with our calculations based on the stochastic modelling approach. 
Figure 4: Primitive equation model based nondimensional subgrid-scale parameterisations. Shown are (a) drain dissipation, (b) backscatter dissipation and (c) net dissipation for vorticity, (d) net dissipation for divergence.

(a)

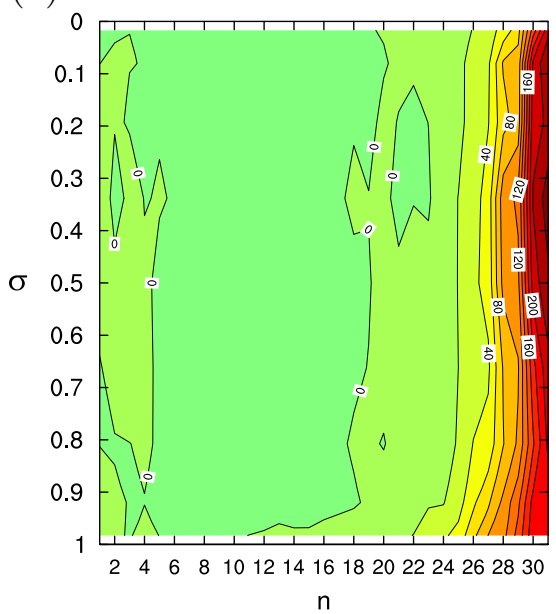

(c)

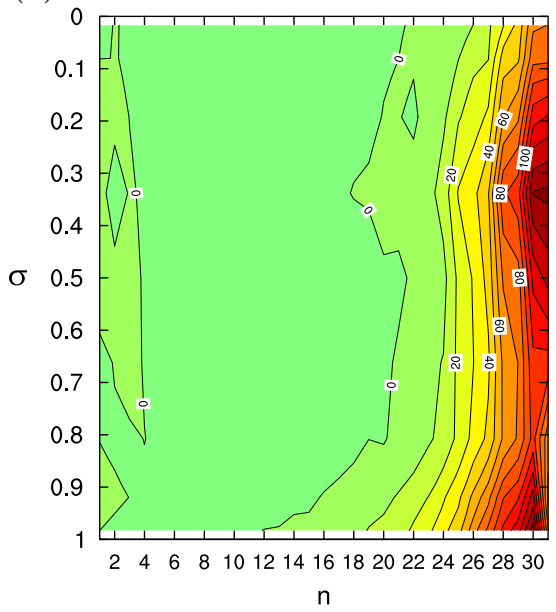

(b)

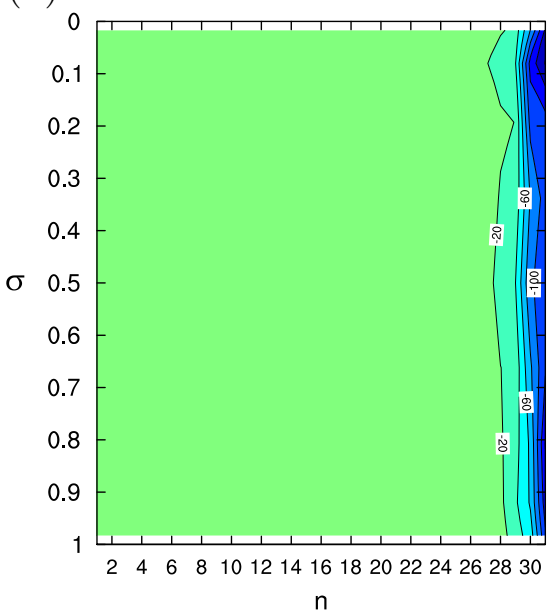

(d)

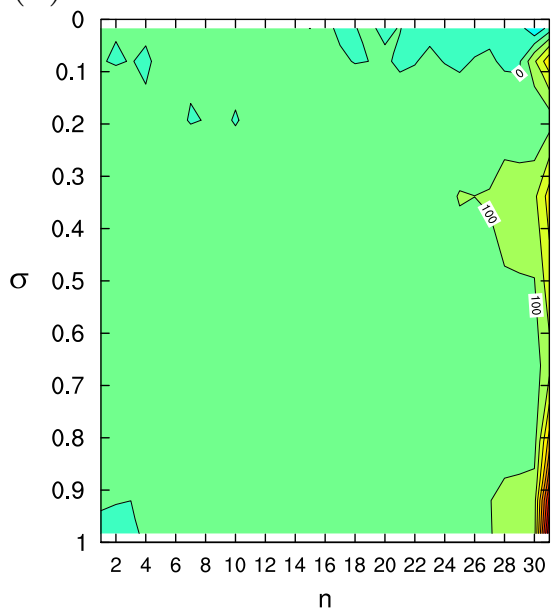


Figure 5: Primitive equation model based nondimensional subgrid-scale parameterisations. Shown are (a) net dissipation for temperature as function of $\sigma$ level and total wavenumber $n$ and (b) net dissipation for surface pressure as function of $n$.

(a)

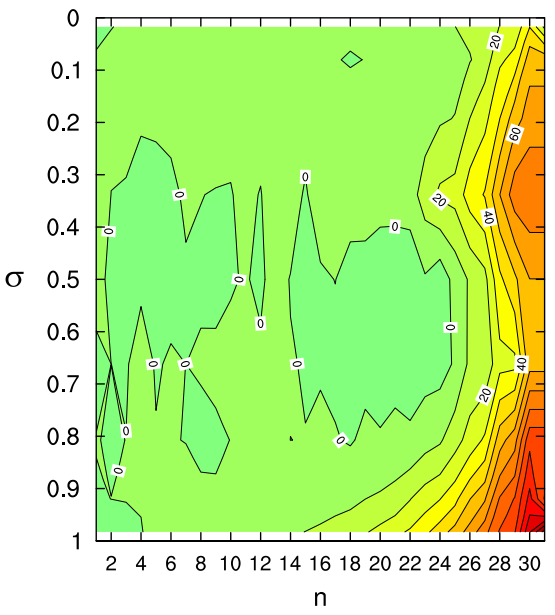

(b)

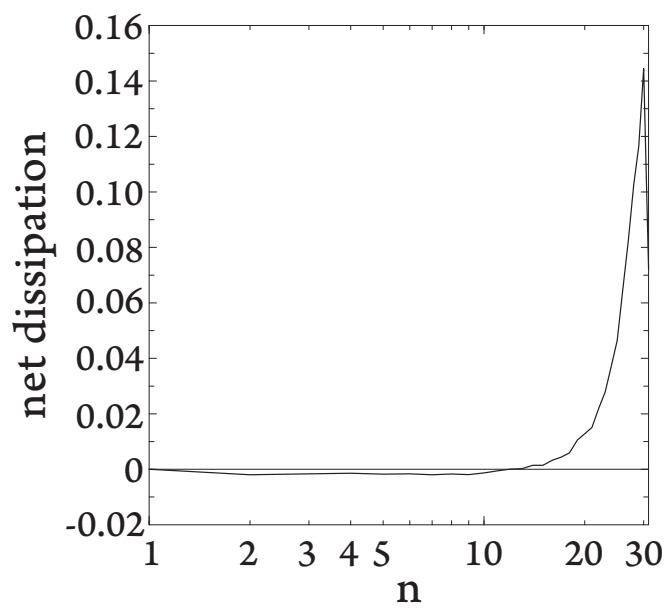

In summary, in broad terms, the dissipation functions $\mathrm{g}$ or $\mathrm{g}^{+}$, scaled as discussed above, represent the effect of subgrid scale eddies on the vorticity, divergence and the temperature at the retained scales.

\section{AGCM simulations with subgrid model}

For the simulations described in this section, we again use the primitive equation CSIRO AGCM detailed by McGregor et al. [9]. However, instead of the simplified relaxation physics employed in Section 4, the simulations are performed with a comprehensive set of dynamical and thermodynamical processes. The AGCM includes radiation and precipitation, snow, sea-ice and cloud amounts that are determined through prognostic equations, and with 
variability from diurnal to seasonal time scales. An interactive land-surface scheme is used and sea-surface temperatures are prescribed monthly and interpolated in time. The vertical coordinate is the $\sigma$ coordinate described in Section 2 where the generic form of the spectral equations are also outlined for the 9-level model. Again, the horizontal resolution corresponds to triangular T63 truncation. The model is integrated with a semi-implicit leap-frog scheme and employs an Asselin time filter and time steps varying between 4 and 15 minutes.

Dissipation is included as shown in equation (1) and the standard formulation [10] used in the control simulation is specified by the heuristic form

$$
D_{0}^{\bullet}(n, \sigma)=k_{0}^{\bullet} n(n+1) \Delta(n),
$$

where $\Delta(n)$ is unity if $60 \leqslant n \leqslant 63$ and otherwise is zero. Also, $k_{0}^{\top}=k_{0}^{\zeta}$ and $\kappa_{0}^{D}=4 \kappa_{0}^{\zeta}$ for numerical stability in the stratosphere. The standard diffusion parameterisation for the control simulation also uses $k_{0}^{\zeta} a_{e}^{2}=6.25 \times 10^{4} \mathrm{~m}^{2} \mathrm{~s}^{-1}$.

The AGCM was integrated for 50 years with climatological sea surface temperatures to reach its converged climate state and the control state was specified by a further 10 year integration from which the average flow properties and kinetic energy spectra were determined for each month of the annual cycle.

Perhaps the clearest indication of a problem with the control simulation using the heuristic standard diffusion parameterisation is seen from the kinetic energy spectra (vertically integrated over the $\sigma$ levels) shown in Figure 6. This depicts the January total wavenumber kinetic energy spectrum $e(n)$ in $\mathrm{m}^{2} \mathrm{~s}^{-2}$ and the spectrum \pm standard deviation for the control AGCM simulation averaged over 10 years. Figure 6 shows that the control simulation underestimates the kinetic energy at wavenumbers between 4 and 25 compared with the observed spectrum for January 1979. On the other hand, at small scales the kinetic energy is too large compared with observations and does not follow the $\mathrm{n}^{-3}$ inertial range power law.

The discrepancies seen in the kinetic energy spectra in Figure 6 with the standard heuristic diffusion parameterisation are reflected in the flow fields 
Figure 6: Kinetic energy spectra $e(n)\left(\mathrm{m}^{2} \mathrm{~s}^{-2}\right)$ for control AGCM simulation for January (thick solid) and $e(n) \pm$ standard deviation for control simulation (dashed). Also shown are $\boldsymbol{e}(\mathrm{n})$ for January 1979 (thin solid).

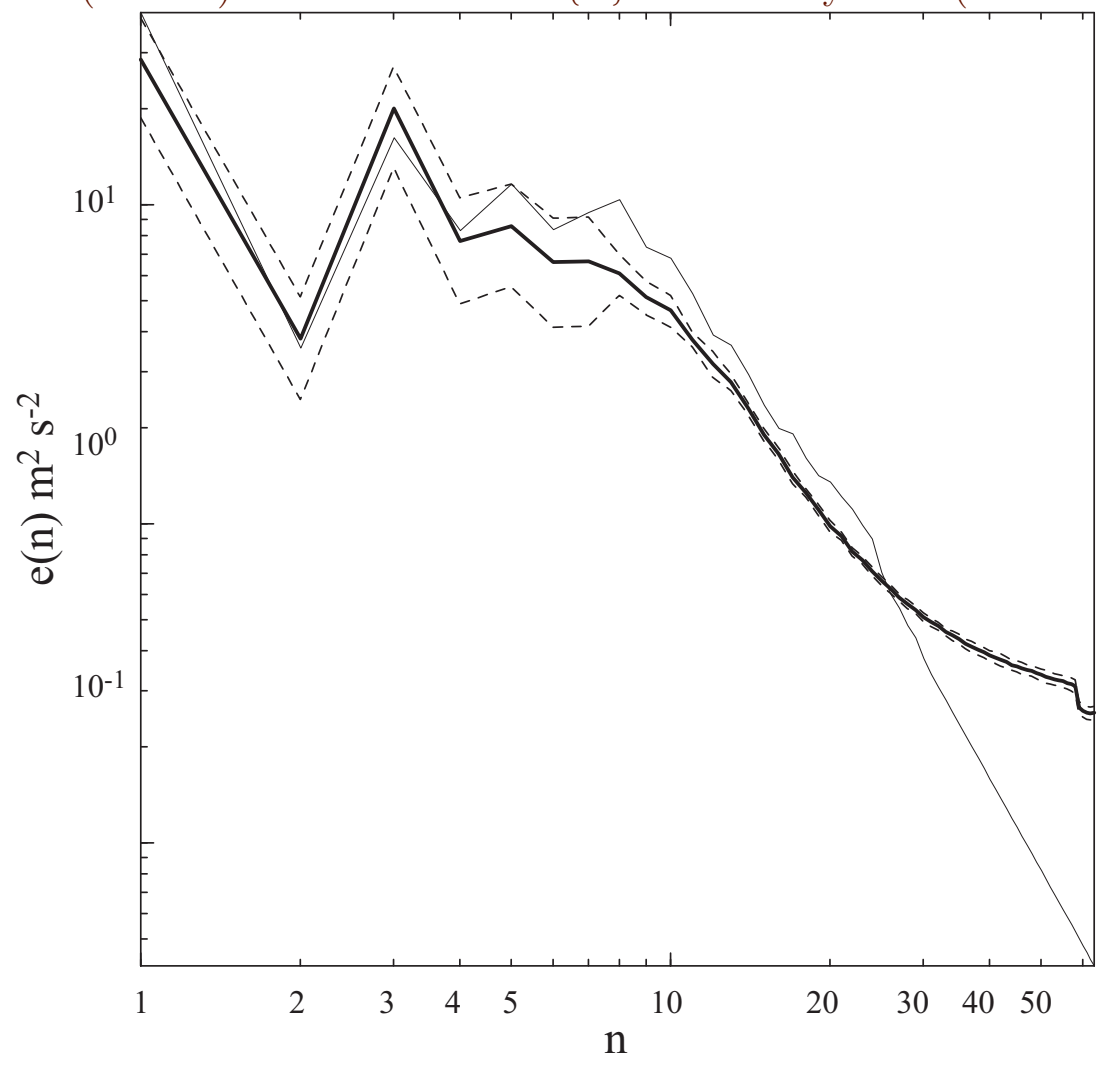


and particularly in the simulations of the zonal winds shown in Figure 3(c). Here the zonal winds averaged over 10 years for January are compared with reanalyses, in Figure 3(b), based on the European Center for Medium Weather Forecasting (ECMWF) dataset for 1985-1992. While the main feature of the circulation are reproduced by the control simulation, the jetstream maxima are underestimated and the northern hemisphere stratospheric jet is too weak. We find similar deficiencies with the control simulations compared with reanalysed observations in other months (not shown).

As noted in Section 4, the vertically integrated dissipation functions based on the primitive equations correspond closely to $g\left(n / n_{*}\right)$, or $g^{+}\left(n / n_{*}\right)$, since the magnitude of the negative contribution is quite small or non existent for divergence and temperature. We employ the dissipation representation given in equation (8) with $v_{0}^{\zeta} \mathrm{a}_{e}^{2}=\mathrm{S} \times 4.92 \times 10^{4} \mathrm{~m}^{2} \mathrm{~s}^{-1}$ for $\mathrm{S}=1,5,10$ using the dissipation function $\mathrm{g}^{+}$(denoted runs $\mathrm{S1}$, S5 and S10) and for $S=5$ with dissipation function $\mathrm{g}$ (denoted run N5). A scaling factor of $S=1$ at T31 corresponds to the calculated eddy viscosity strength for barotropic flow at $500 \mathrm{hPa}$ [3]. At $300 \mathrm{hPa}$ a scaling factor of $S \approx 5$ at T63 would appear to be most appropriate although we find that $S=10$ better compensates for the weaker zonal winds in the control simulation Figure 3(c).

The vertically integrated total wavenumber kinetic energy spectra $e(n)$ in $\mathrm{m}^{2} \mathrm{~s}^{-2}$ are shown in Figure 7 for January for the S1, S5, S10 and N5 runs. The results are averaged over ten Januaries. Also shown is the observed spectrum for January 1979 and we see that the S5 and S10 spectra closely agree with the observations at most scales. The N5 spectrum also closely follows the January observed spectrum at large scales with wavenumbers less than 25 but with some over estimation at higher wavenumbers. The S1 spectrum has too weak dissipation at T63 at the end of the spectrum and there is an underestimation of kinetic energy at intermediate scales, as expected [5]. In other months the S5 and S10 runs also have spectra with the correct $\mathrm{n}^{-3}$ inertial ranges (not shown).

Figure 3(d) shows the January zonal winds averaged over 10 years for 
Figure 7: Kinetic energy spectra $e(n) m^{2} s^{-2}$ for $S 1$ run (thick short dashed), N5 run (thin long dashed), S5 $\times 10^{-1}$ (thin short dashed) and S10 run $\times 10^{-1}$ (thick solid) all for January. Also shown are $e(n)$ for January 1979 (thin solid).

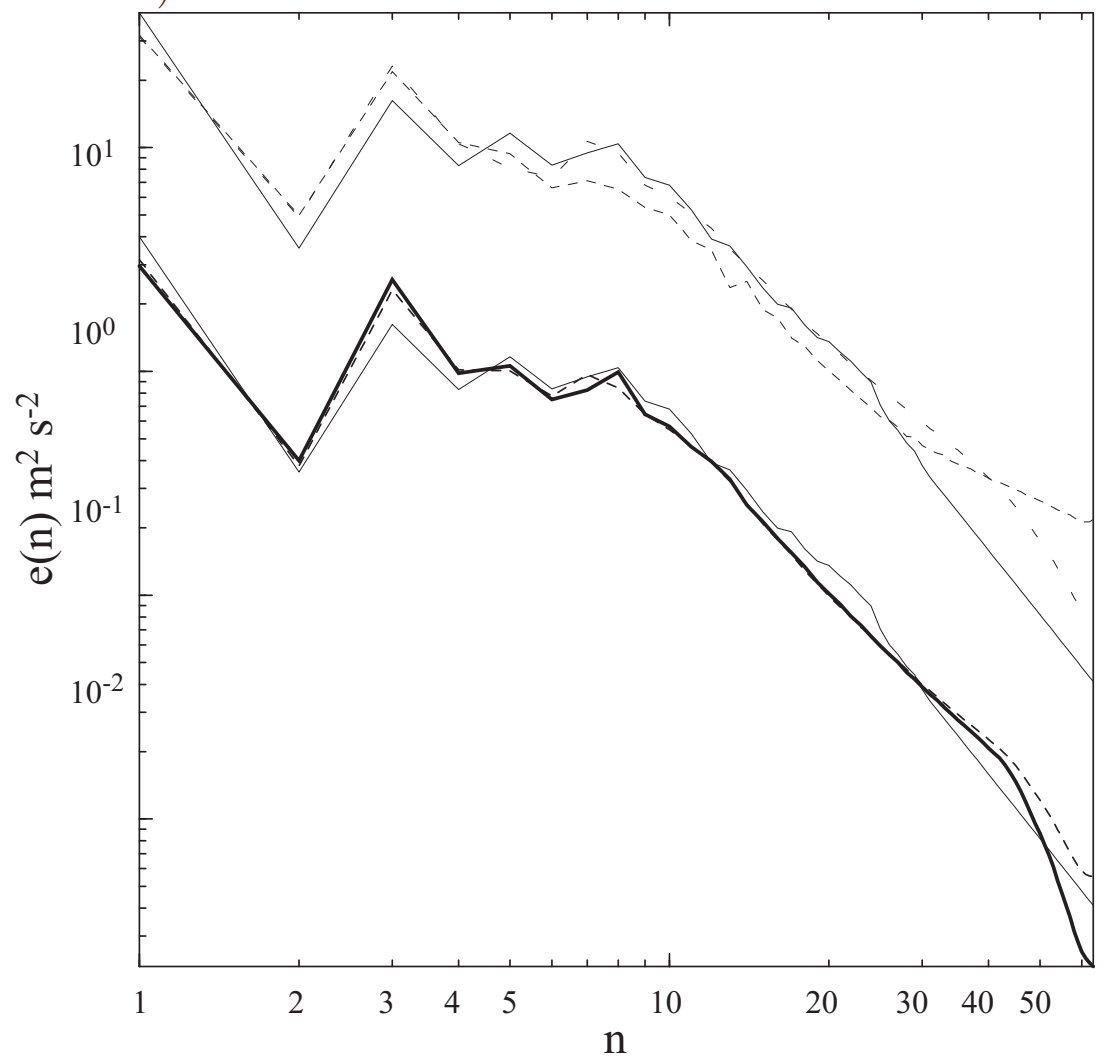


the S10 run. The peak zonal winds for the Northern Hemisphere tropospheric jet core are around $5 \mathrm{~ms}^{-1}$ stronger than in the control run with the standard diffusion parameterisation equation (9) and in better agreement with the observations (Figure 3(b)). Similarly, in the Southern Hemisphere the tropospheric jet core is also more equatorward and strengthened by nearly $5 \mathrm{~ms}^{-1}$ compared with the control. The zonal wind simulation in the S5 and N5 runs are very similar both for January and for the other months (not shown). Again, the differences between S10 and the control and S5 and the control are quite similar but the magnitudes are larger in the $S 10$ case. This suggests that the stronger diffusion may be compensating for other AGCM deficiencies.

\section{Conclusions}

Dynamical and thermodynamical subgrid-scale parameterisations that represent the effects of unresolved eddies on the resolved scales of vorticity, divergence, temperature and surface pressure, were calculated from simulations with a nine-level primitive equation model. The stochastic modelling method of Frederiksen and Kepert [3] was employed in simulations of typical atmospheric flows. The drain dissipation, backscatter dissipation and net dissipation functions were calculated for each of the dynamical fields as functions of level and total wavenumber. When vertically integrated, the net dissipation functions have very similar structures and magnitudes to the barotropic case with a cusp near the largest wavenumbers. However, the strength of the divergence dissipation is around four times stronger than for the other fields. The vertically integrated eddy dissipation and diffusion parameterisations were applied to the same AGCM including comprehensive physical processes. Compared with the control simulation, using heuristic diffusion functions, the simulations with our derived parameterisations improve the kinetic energy spectra and the zonal wind strength by as much as $5 \mathrm{~ms}^{-1}$. 


\section{References}

[1] J. S. Frederiksen. Self-energy closure for inhomogeneous turbulence and subgrid modelling. Entropy 14:769-799, 2012. doi:10.3390/e14040769 C86

[2] J. S. Frederiksen and A. G. Davies. Eddy viscosity and stochastic backscatter parameterizations on the sphere for atmospheric circulation models. J. Atmos. Sci 54:2475-2492, 1997. doi:10.1175/1520-0469(1997)054<2475:EVASBP > 2.0.CO;2 C87

[3] J. S. Frederiksen and S. M. Kepert. Dynamical subgrid-scale parameterizations from direct numerical simulations. J. Atmos. Sci. 63:3006-3019, 2006. doi:10.1175/JAS3795.1 C84, C86, C87, C91, C96, C98

[4] J. S. Frederiksen, T. J. O'Kane and M. J. Zidikheri. Stochastic subgrid parameterizations for atmospheric and oceanic flows. Phys. Scripta 85:068202, 2012. doi:10.1088/0031-8949/85/06/068202 C84

[5] J. S. Frederiksen, M. R. Dix and S. M. Kepert. Systematic energy errors and the tendency toward canonical equilibrium in atmospheric circulation models. J. Atmos. Sci. 53:887-904, 1996. doi:10.1175/1520-0469(1996)053<0887:SEEATT>2.0.CO;2 C84, C96

[6] I. M. Held, and M. J. Suarez. A proposal for the intercomparison of the dynamical cores of atmospheric general circulation models. Bull. Am. Met. Soc. 75:1825-1830, 1994. doi:10.1175/1520-0477(1994)075<1825:APFTIO>2.0.CO;2 C87

[7] V. Kitsios, J. S. Frederiksen and M. J. Zidikheri. Scaling laws for parameterisations of subgrid eddy-eddy interactions in simulations of oceanic circulations. Ocean Model. 68:88-105, 2013. doi:10.1016/j.ocemod.2013.05.001 C84, C87 
[8] V. Kitsios, J. A. Sillero, J. S. Frederiksen and J. Soria. Proposed stochastic parameterisations of subgrid turbulence in large eddy simulations of turbulent channel flow. J. Turbulence 16:729-741, 2015. doi:10.1080/14685248.2015.1026970 C84

[9] J. L. McGregor, H. B. Gordon, I. G. Watterson, M. R. Dix and L. D. Rotstayn. The CSIRO 9-level atmospheric general circulation model. CSIRO Division of Atmospheric Research, Technical Report 26, 1993. https://publications. csiro.au/rpr/pub?list=BRO\&pid=procite: d73112b4-4d15-481b-84c5-f488501e7e07 C85, C93

[10] I. N. Smith, M. R. Dix and R. J. Allen. The effect of greenhouse SSTs on ENSO simulations with an AGCM. J. Climate 10:342-352, 1997. doi:10.1175/1520-0442(1997)010<0342:TEOGSO > 2.0.CO;2 C94

\section{Author addresses}

1. J. S. Frederiksen, CSIRO Oceans and Atmosphere, Aspendale 3195, Australia. mailto: Jorgen.Frederiksen@csiro.au

2. M. R. Dix, CSIRO Oceans and Atmosphere, Aspendale 3195, Australia.

3. S. L. Osbrough, CSIRO Oceans and Atmosphere, Aspendale 3195, Australia.

4. V. Kitsios, CSIRO Oceans and Atmosphere, Aspendale 3195, Australia. 